\title{
A NEW COMPOSITE-SAMPLE METHOD FOR ORIENTATION-DISTRIBUTION ANALYSIS
}

\author{
P. R. MORRIS, $\dagger$ R. E. HOOK, $\ddagger$ and G. W. WHELAN $\ddagger$ \\ $\dagger 1276$ Oakmont Dr., Hamilton, OH 45013, USA \\ $\ddagger$ Research and Technology, Armco Inc., Middletown, OH 45043, USA
}

(Received August 30, 1990)

\begin{abstract}
A new composite-sample method is suggested for crystallite orientation distribution analysis. The proposed method entails preparation of composites such that the surface to be examined is perpendicular to the rolling direction. The suggested reference frame is (-ND, TD, RD) for Roe's method. This choice simplifies expression of the results with respect to the conventional (RD, TD, ND) frame. A novel technique employing laser welding on three surfaces is used to bond adjacent sheet layers. The proposed composite-sample can be more easily and accurately constructed. It requires only about 12 percent of the material needed for Lopata and Kula's method. Existing programs for incomplete pole-figures have been modified to permit application of the new method. The method is expected to be statistically advantageous where materials develop "pancake"- or acicular-shaped grains. The method is illustrated for a deep-drawing aluminum-killed sheet steel, and results are compared with those obtained with a conventional sheet-sample.
\end{abstract}

KEY WORDS Composite sample, ODF analysis.

\section{INTRODUCTION}

A least-squares procedure for determination of coefficients of the generalized spherical harmonic expansion of the crystallite orientation distribution (ODF) from incomplete pole-figures was first proposed by Bunge (1969). Prior to reduction of this idea to practice by Pospeich and Jura (1974) and by Morris (1975), ODF determination by polycrystal X-ray diffraction experiments usually entailed a combination of transmission and back-reflection measurements, or preparation of the type of composite-sample suggested by Lopata and Kula (1962). Alternative treatments of incomplete pole-figure data have been suggested by Kern and Bergmann (1979), van Houtte (1980, 1984), Humbert and Bergmann (1980), Liang et al. (1981, 1983), and Dahms and Bunge (1986, 1987, 1988). The use of incomplete pole-figure data simplifies sample preparation and allows investigation of texture as a function of position by removal of successive surface layers between measurements.

During rolling and annealing, some materials develop "pancake"- or acicularshaped grains. In these cases, measurements on surfaces perpendicular to the rolling direction are statistically advantageous with respect to those on surfaces perpendicular to the normal direction. The previously-cited methods for using incomplete pole-figure data, in conjunction with relations (Roe, 1965; Bunge, 1982) for series coefficients for a rotated reference frame, allow the results of the measurements on other surfaces to be expressed with respect to the conventional reference frame. 


\section{PROPOSED COMPOSITE-SAMPLE METHOD}

For sheet, we propose the use of a composite-sample in which the surface to be examined is perpendicular to the rolling direction (RD), i.e. the sample surface is the normal direction (ND), transverse direction (TD) plane. The suggested reference frames are (ND, TD, -RD) with $\alpha=0, \beta=\pi / 2, \gamma=0$; or $(-\mathrm{ND}$, TD, RD) $\alpha=\pi, \beta=\pi / 2, \gamma=\pi$ for Roe's method. For these choices the factors $\exp (-i p \alpha)$ and $\exp (-i p \gamma)$ are real, simplifying application of Roe's Eq. (17). This type of composite-sample can be more easily and accurately prepared, needs much less material than that of Lopata and Kula, and requires no assumptions concerning the presence of sample-symmetry operations.

An alternative composite-sample in which the surface to be examined is perpendicular to the transverse direction (TD), i.e. the sample surface is the normal direction (ND), rolling direction (RD) plane, employs the reference frame (ND, RD, TD) with $\alpha=0, \beta=\pi / 2, \gamma=\pi / 2$ for Roe's method.

\section{COMPOSITE-SAMPLE CONSTRUCTION}

Figure 1 is a schematic drawing which shows the relationships between the physical reference frames of a (RD, TD, ND) sheet-sample, a (-ND, TD, RD)

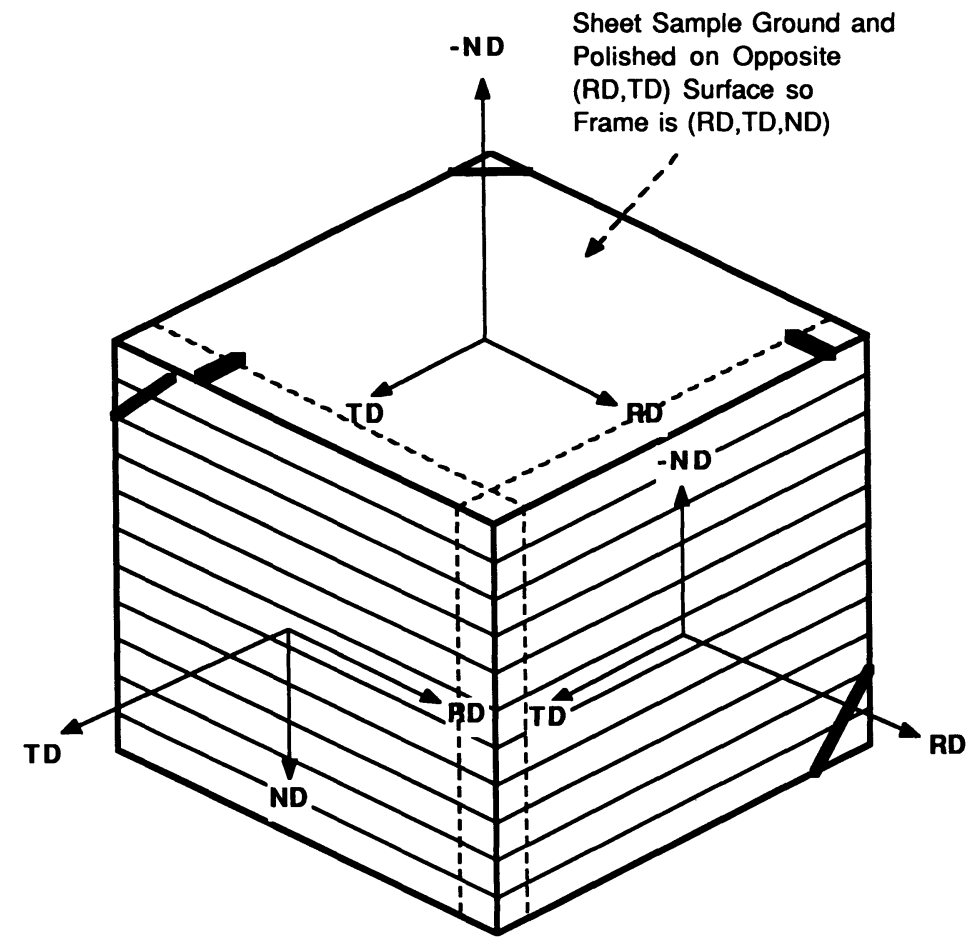

Figure 1 Relationship between reference frames of sheet and composite-samples and orientation of samples for X-ray data collection so that sense of frames is always preserved among the three frames. Corner mark is always placed to upper-left when viewing data collection surface of (RD, TD, ND) sheet-sample, (-ND, TD, RD) composite-sample, and (ND, RD, TD) composite-sample. 
composite-sample, and a (ND, RD, TD) composite-sample such that the sense of the directions relating any given sample to the other two is preserved.

Figure 2 illustrates the method for construction of a composite-sample having a (-ND, TD, RD) reference frame. A sheet-sample is shear cut into coupons of the desired size after marking the RD as shown in Figure 2(a). The coupons are then stacked in such a manner so as to conserve the sense of the RD by overlaying the ink marked ends on each coupon, as shown in Figure 2(b). The stack is then compressed in a vise to hold the stack for welding. In our case we chose to laser weld the stack. Other welding methods would also be acceptable as long as they are not invasive, i.e. they do not input heat to the degree that the structure of the sample is altered at the location where data is to be collected. The weld pass line locations are shown in Figure 2(c). In our case, the width of the weld fusion zones was $0.4 \mathrm{~mm}$, while the depth was $1.6 \mathrm{~mm}$. These are adjustable parameters, as long as the welds are not invasive to the data-collection surface. Figure 3 shows an as-welded (ND, RD, TD) composite-sample, also investigated, which was prepared in a similar way, (see Figure 1).

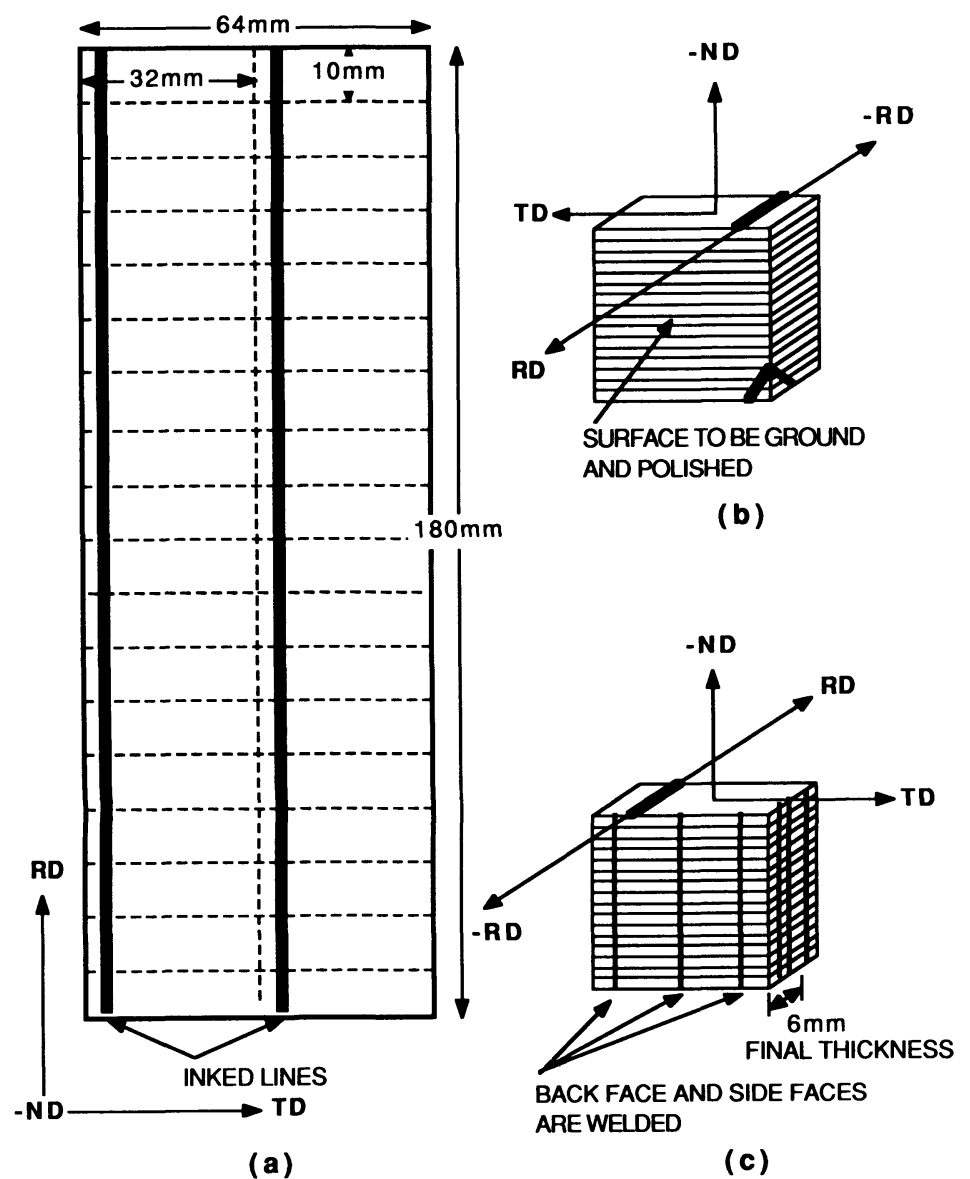

Figure 2 Sample preparation method for (-ND, TD, RD) composite-sample. 


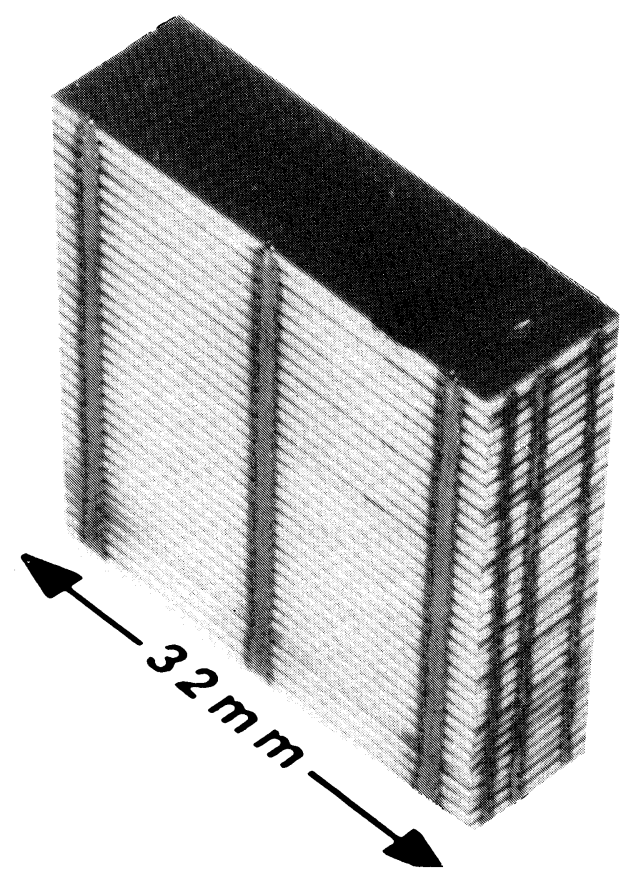

Figure 3 As-welded (ND, RD, TD) composite-sample. Inter-lamellar spacing appears accentuated because coupon edges are chamfered to remove shear burrs.

The composite-samples were then reduced in thickness (the dimension parallel to the RD for the sample shown in Figure 2) from $10 \mathrm{~mm}$ to $6 \mathrm{~mm}$ using a surface grinder. This thickness was the maximum which the X-ray goniometer can accept in our case. The coupons were shear cut to a $10 \mathrm{~mm}$ dimension initially to facilitate accuracy in cutting and ease in handling, but this size selection is arbitrary. The sample was then hand polished using standard metallographic sample preparation methods, through 14 micron wet silicon carbide papers. At this point the coupons were in such intimate contact at the plane of polish (the -ND, TD plane) detecting that the sample was actually a composite was difficult, even at a magnification of $30 \times$.

Before collecting X-ray data from the (-ND, TD, RD) composite-sample, $0.025 \mathrm{~mm}$ was removed from the surface to be irradiated by chemical etching in a $1: 1$ mixture of 70 percent phosphoric acid: 30 percent hydrogen peroxide at ambient temperature. This was sufficient to remove any residual cold work. After etching, the composite nature of the sample was discernable as a result of attack by the etchant of the coupon edges at the coupon to coupon interfaces. This localized preferential etching resulted in the formation of $\mathrm{V}$-shaped grooves in the composite-sample surface, (-ND, TD) plane, running parallel to TD. The groves had a spacing equal to the coupon thickness of $0.94 \mathrm{~mm}$, a half-width of $0.07 \mathrm{~mm}$, and a depth of $0.14 \mathrm{~mm}$. As a result, the ratio of the grooved area to the total area for the surface plane of the composite-sample to be irradiated was 0.15 . In the worst case, this means that data may not be collected, when the incident X-ray beam is normal to the grooves at $\eta=0$ and $180 \mathrm{deg}$, from gains at 


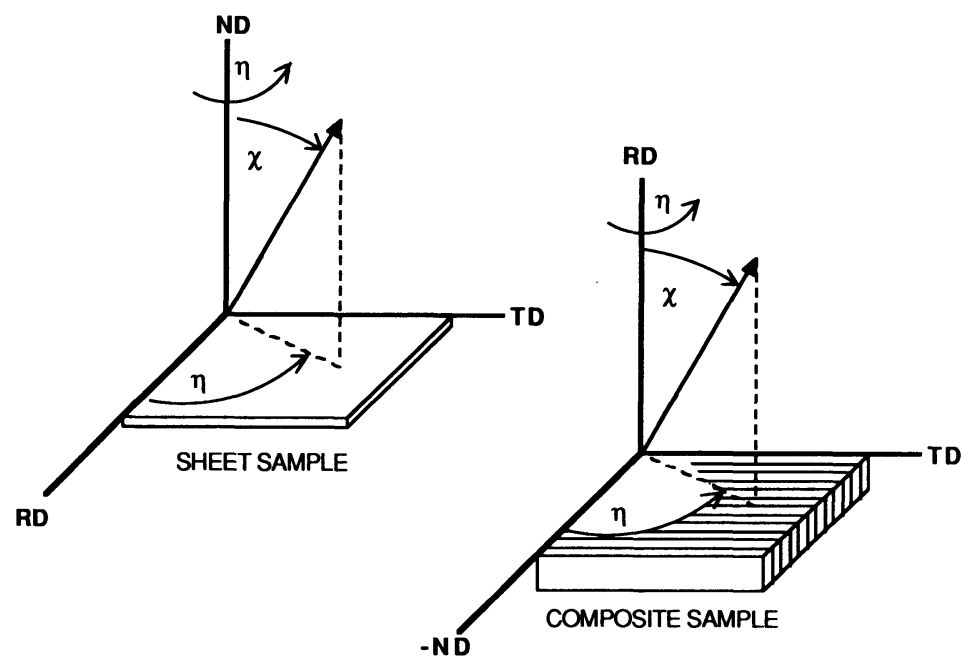

Figure 4 Relationship between sample's physical coordinates and the azmuthal and longitudinal angles used to collect $\mathrm{X}$-ray incomplete pole-figure data.

the coupon's rolling surfaces to a depth of 7.5 percent of the total sheet thickness at each surface. The possible effect of the grooves on data acquisition is being investigated. If found to not be negligible, methods to remove cold work which avoid grooving will have to be developed.

The relationship between the $\chi$ and $\eta$ angles for X-ray data collection and the orientation of the (RD, TD, ND) sheet-sample and the (-ND, TD, RD) composite-sample is shown in Figure 4.

Before collecting X-ray data from the second type of (ND, RD, TD) composite-sample, $0.0125 \mathrm{~mm}$ was removed from the surface to be irradiated by chemical etching, one-half the amount removed for the (-ND, TD, RD) sample. The results indicated this amount of surface removal was sufficient to eliminate any influence of cold work. While the grooving was not eliminated, it was reduced from 15 percent of the area to 6 percent of the area. Thus in the worst case, grains at the rolling surfaces of each coupon making up the composite amounting to 3 percent of the sheet thickness at each surface may not be collected.

\section{GEOMETRICAL FACTORS}

The reduced amount of material required for the proposed composite-sample, compared to that of Lopata and Kula may be an advantage. For example, for laboratory produced sheet the width is usually limited to the order of $100 \mathrm{~mm}$. In this case, the proposed sample requires only about 12 percent of the material required for the Lopata and Kula sample.

In the application of our method to an aluminum-killed sheet steel with a "pancake" grain structure (see Figure 5) the average grain aspect ratio when viewed on a plane perpendicular to the ND was $(R D: T D=1.25: 1)$, when 


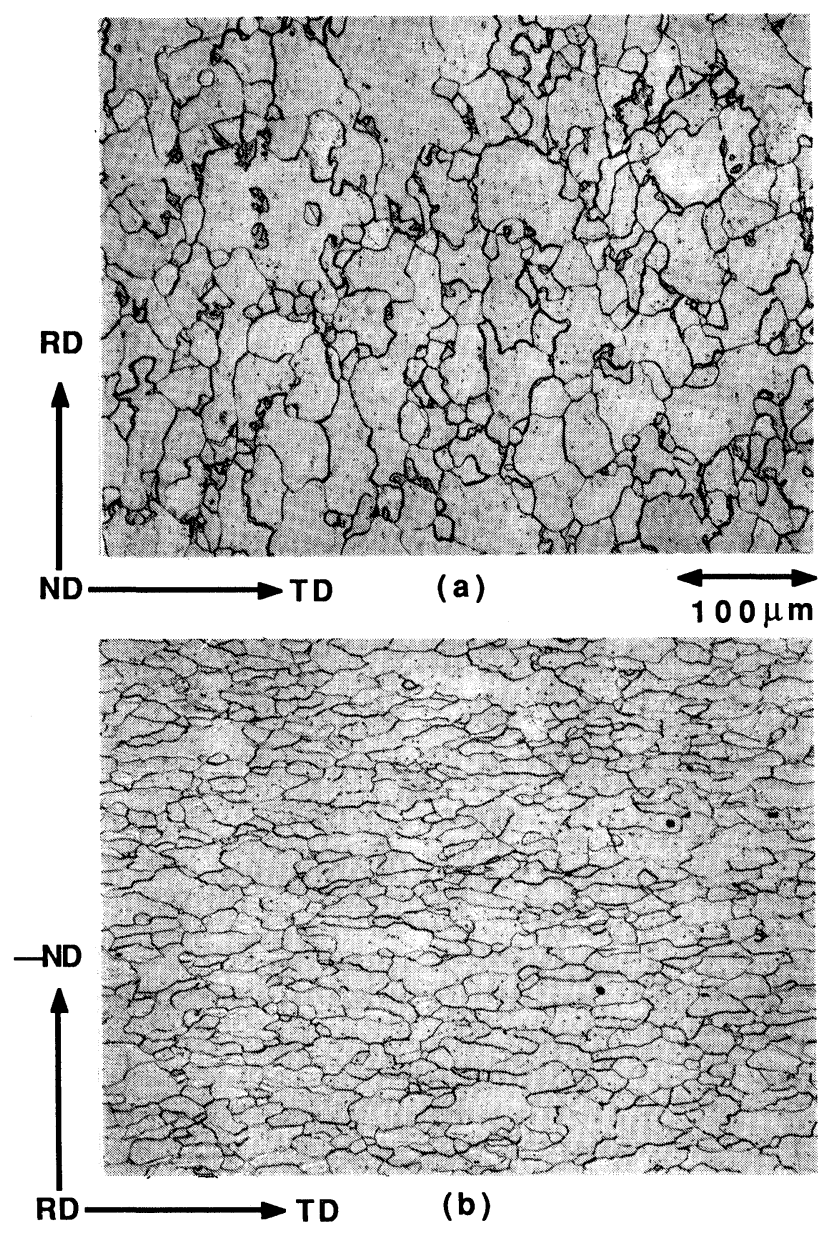

Figure 5 Grain structure of (a) sheet-sample for (RD, TD) plane and (b) composite-sample for (-ND, TD) plane.

viewed on a plane perpendicular to the RD the ratio was $(T D: N D=1.76: 1)$ From a determination of the number of grains per unit area presented for both views, it was ascertained that the ratio of the number of grains sampled during $\mathrm{X}$-ray data collection, composite-sample (-ND, TD, RD) to sheet-sample (RD, $\mathrm{TD}, \mathrm{ND})=2.2: 1$. Based on our measurements of the surface area irradiated at $\chi=70 \mathrm{deg}$ and the number of grains per unit area, we determined approximately 158,000 grains of the (-ND, TD, RD) composite-sample and 71,000 grains of the (RD, TD, ND) sheet-sample were irradiated. Similarly, for the (ND, RD, TD) composite-sample the average grain aspect ratio when viewed on a plane perpendicular to the TD was (RD : ND =2.2:1), and approximately 125,000 grains were irradiated. Therefore, in these cases the (-ND, TD, RD) compositesample provided for measuring the largest grain population. 


\section{EXPERIMENTAL RESULTS}

Existing computer programs have been modified to permit collection of data for a composite samples with (-ND, TD, RD) and (ND, RD, TD) reference frames and expression of results with respect to the conventional (RD, TD, ND) sheet-sample reference frame. Figure 6(a), (b), and (c) illustrate the $\phi=45 \mathrm{deg}$ sections of a 22nd order expansion of the ODF for a cold-rolled, batch-annealed, deep-drawing aluminum-killed sheet steel, obtained (a) with the proposed (-ND, TD, RD) composite-sample, (b) with a (ND, RD, TD) compositesample, and (c) with a conventional (RD, TD, ND) sheet-sample at the

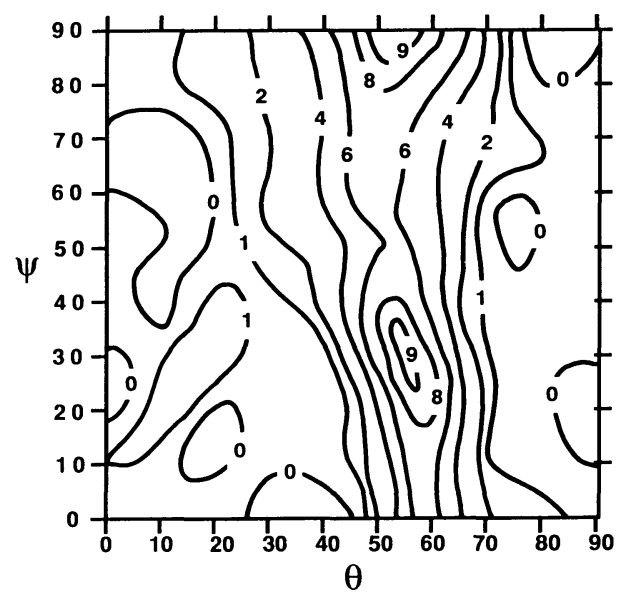

(a)

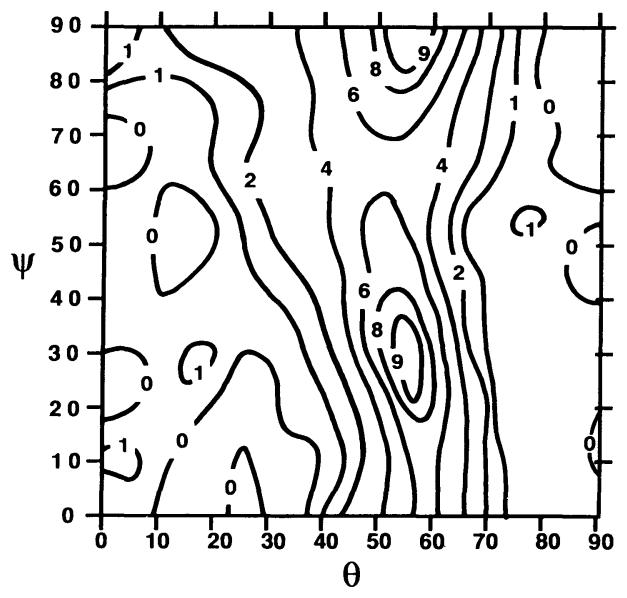

(b)

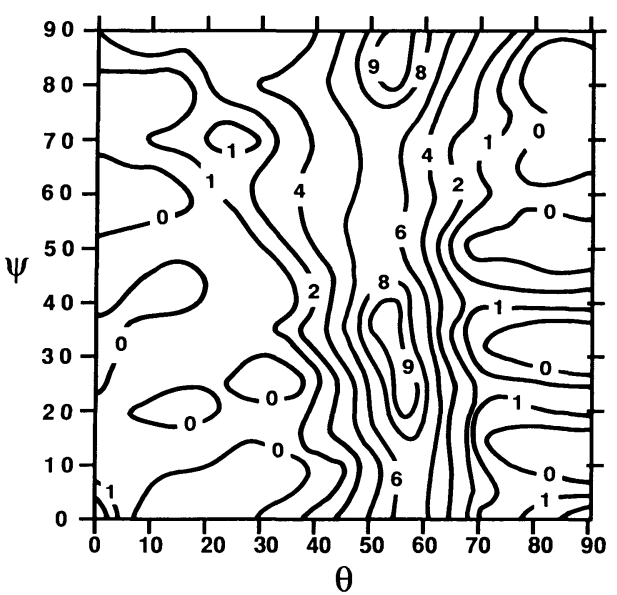

(c)

Figure 6 (a) $\phi=45 \mathrm{deg}$ section of the ODF of an aluminum-killed steel sheet determined using a (-ND, TD, RD) composite-sample. (b) $\phi=45 \mathrm{deg}$ section of the ODF of an aluminum-killed steel sheet determined using a (ND, RD, TD) composite-sample. (c) $\phi=45 \mathrm{deg}$ section of the ODF of an aluminum-killed steel sheet determined using a (RD, TD, ND) sheet-sample. 
one-quarter thickness position. Orthorhombic sample symmetry has been imposed, and only the coefficients with $L$ even have been used. Maxima cor-

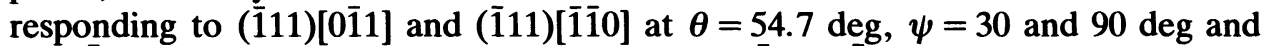
the $(\overline{1} 11)[u v w]$ orientation tube running from $(\overline{1} 11)[1 \overline{1} 2]$ at $\theta=54.7 \mathrm{deg}, \psi=0$ deg to $(\overline{1} 11)[\overline{1} \overline{1} 0]$ have similar values and contours in all sections. The agreement is remarkable, since we are not necessarily dealing with geometrically-equivalent grain populations in the composite and sheet samples.

\section{Acknowledgements}

The authors thank Armco Inc. for permission to publish. We also thank William W. Nagle, Process Control, Armco Research Center for conducting the laser welding used in constructing the composite-samples.

\section{References}

Bunge, H. J. (1969). Mathematische Methoden der Texturanalyse. Akademie-Verlag, Berlin.

Bunge, H. J. (1982). Texture analysis in Materials Science. Butterworths, London, Eq. (8.16).

Dahms, M. \& Bunge, H. J. (1986). Textures and Microstructures 6, 167-79.

Dahms, M. \& Bunge, H. J. (1987). Textures and Microstructures 7, 171-85.

Dahms, M. \& Bunge, H. J. (1988). Textures and Microstructures 10, 21-35.

van Houtte, P. (1980). Mat. Sci. Eng. 43, 7-11.

van Houtte, P. (1984). Textures and Microstructures 6, 137-61.

Humbert, M. \& Bergmann, H. W. (1980). J. Appl. Cryst. 13, 500-4.

Kern, R. \& Bergmann, H. W. (1979). In: Textures of Materials, Eds. G. Gottstein and K. Luke, Springer-Verlag, Berlin.

Liang, Z., Xu, J. \& Wang, F. (1981). Proc. ICOTOM 6, Tokyo, 1259-64.

Liang, Z., Xu, J. \& Wang, F. (1983). Mat. Sci. Eng. 60, 59-63.

Lopata, S. L. \& Kula, E. B. (1962). Trans. Met. Soc. AIME 224, 865-6.

Morris, P. R. (1975). Adv. in X-ray Anal. 18, Plenum Press, New York, 514-34.

Pospiech, J. \& Jura, J. (1974). Z. Metallkde. 65, 324-30.

Roe, R. J. (1965). J. Appl. Phys. 36, 2024-31, Eq. (17). 